\title{
A Fault Diagnosis Method for Oil Well Pump Using Radial Basis Function Neural Network Combined with Modified Genetic Algorithm
}

\author{
Deliang Yu, ${ }^{1,2}$ Yanmei Li, ${ }^{1}$ Hao Sun, ${ }^{1}$ Yulong Ren, ${ }^{1}$ Yongming Zhang, ${ }^{3}$ and Weigui Qi ${ }^{4}$ \\ ${ }^{1}$ Harbin University of Science and Technology, Harbin 150001, China \\ ${ }^{2}$ Research Institute of Oil Production Engineering, Daqing Oilfield Company, Daqing 163000, China \\ ${ }^{3}$ Tongji University, Shanghai 200092, China \\ ${ }^{4}$ Harbin Institute of Technology, Harbin 150001, China
}

Correspondence should be addressed to Deliang Yu; yudeliang1032@163.com

Received 26 April 2017; Revised 16 August 2017; Accepted 29 August 2017; Published 8 October 2017

Academic Editor: Manuel Pineda-Sanchez

Copyright (C) 2017 Deliang Yu et al. This is an open access article distributed under the Creative Commons Attribution License, which permits unrestricted use, distribution, and reproduction in any medium, provided the original work is properly cited.

\begin{abstract}
This paper presents a new method to diagnose oil well pump faults using a modified radial basis function neural network. With the development of submersible linear motor technology, rodless pumping units have been widely used in oil exploration. However, the ground indicator diagram method cannot be used to diagnose the working conditions of rodless pumping units because it is based on the load change of the polished rod suspension point and its displacement. To solve this problem, this paper presents a new method that is applicable to rodless oil pumps. The advantage of this new method is its use of a simple feature extraction method and advanced genetic algorithm to optimize the threshold and weight of the RBF neural network. In this paper, we extract the characteristic value from the operation parameters of the submersible linear motor and oil wellhead as the input vector of the fault diagnosis model. Through experimental analysis, the proposed method is proven to have good convergence performance, high accuracy, and high reliability.
\end{abstract}

\section{Introduction}

The special structure of the beam pumping units has many disadvantages, such as huge form, high cost, high energy consumption, and low efficiency. A new rodless oil well pump has been proposed with the development of the submersible linear motor [1]. The submersible linear motor drives the pump plunger directly to move linearly [2]. The new oil well pump has a good application potential because it addresses the disadvantages of the beam pumping units [3].

The structure of the new oil well pump is inverted; that is, the plunger pump is placed on the submersible linear motor [4]. The motor stator is connected to the oil tube and its mover is connected to the travelling valve using the pump plunger. When the mover of linear motor performs a reciprocating motion, the travelling valve is driven to move and transfer liquid of the casing into the pump chamber. Oil is then lifted to the ground wellhead using the tube. Oil extraction efficiency improved greatly when the new oil well pump was used.

Wax deposition, sand sticking, valve leakage, and liquid filling dissatisfaction problems easily occur in oil well pumps because the working conditions of oil wells are abominable. If these problems are not resolved immediately, the reliability and life expectancy of oil well pumps and oil production will decrease.

The ground indicator diagram method is based on the load change of the polished rod suspension point and its displacement. Thus, this paper presents a new method that is applicable to rodless oil well pumps. Genetic algorithm (GA) or the particle swarm optimization (PSO) algorithm is usually combined with neural network and applied in fault diagnosis [5-7]. Intelligent algorithm can help the model find an optimal solution $[8,9]$. However, this method easily encounters problems, such as premature convergence and local optimum [10-12]. To overcome these problems, 


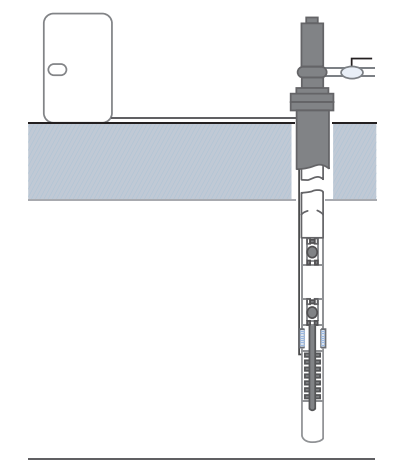

FIGURE 1: Experiment platform.

this paper proposes a fault diagnosis method based on the multiple mutation adaptive genetic algorithm-radial basis function neural network (MMAGA-RBFNN), which is suitable for oil well pumps. The characteristic value from the oil production system should be extracted as the input of the model and the weight and threshold value of RBFNN should be optimized using the MMAGA method according to the structure characteristics of oil production systems and oil well pumps. This model has unique advantages, such as fast convergence rate, high accuracy, and global search ability, and can be used in diagnosing numerous working states.

\section{Feature Extraction}

The working conditions of the motor could be reflected by the operation parameters of the linear motor because the oil well pump is connected directly to the mover of the linear motor. The principle diagram of the oil well pump is shown in Figure 1.

The currents of the linear motor and the loads of the pump differ according to the working conditions of the oil well $[13,14]$. The operating current curves of the linear motor in different working conditions are shown in Figure 2.

Several faults, such as the effect of gases, sand sticking, and wax deposition, can be diagnosed using the current, electric power, and load parameters. Tubing leakage, pump opening, strong mechanical vibration, and motor shock sometimes appear, and faults could not be diagnosed accurately according to these parameters. Therefore, other parameters that reflect the working state of the pump should be considered to increase the diagnostic accuracy rate. In the normal well operating process, the oil and casing pressures of the wellhead are stable. When tubing or pump leakage occurs, the pressure of the wellhead will change accordingly. Therefore, the change in value of oil pressure gauge contributes to further determining the working condition of the well. The temperature of wellhead is also an important parameter for diagnosing the working condition of the oil well because the temperature of wellhead changes with the oil quantity. This paper extracted the characteristic parameters using the multivariate data method, which could reflect the real working condition of the pump and enhance the accuracy rate of the fault diagnosis.

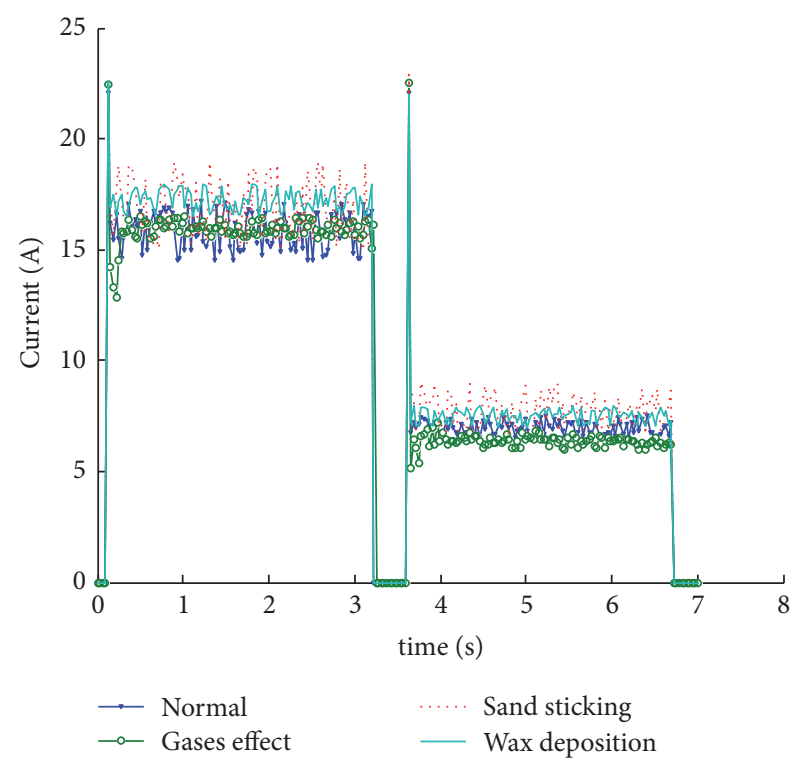

FIGURE 2: Current curves of linear motor.

Through measuring equipment, such as current and pressure sensors, the linear motor can be monitored in real time and the current and load of the motor pump in each cycle can be obtained. At the same time, the oil and casing pressures and temperature of the wellhead can be recorded.

The well data collected are processed as follows:

$$
T_{e}=\frac{1}{l} \sum_{k=1}^{l} T(t),
$$

where $l$ is the number of sampling points in a cycle, $T(t)$ is the real-time temperature of the wellhead, and $T_{e}$ is the mean temperature of the wellhead in a cycle.

$$
F_{o e}=\frac{1}{l} \sum_{k=1}^{l} F_{o}(t),
$$

where $F_{o}(t)$ is the real-time oil pressure of the wellhead and $F_{o e}$ is the mean oil pressure of the wellhead in a cycle.

$$
F_{c e}=\frac{1}{l} \sum_{k=1}^{l} F_{c}(t),
$$

where $F_{c}(t)$ is the real-time casing pressure of the wellhead and $F_{c e}$ is the mean casing pressure of the wellhead in a cycle.

$$
I_{u e}=\frac{1}{m} \sum_{k=1}^{m} i_{u}(t),
$$

where $I_{u e}$ is the mean current on the motor upstroke, $i_{u}(t)$ is the real-time current on the motor upstroke, and $m$ is the number of sampling points on the motor upstroke.

$$
I_{d e}=\frac{1}{n} \sum_{k=1}^{n} i_{d}(t),
$$

where $I_{d e}$ is the mean current on the motor downstroke, $i_{d}(t)$ is the real-time current on the motor downstroke, and 
$n$ is the number of sampling points on the motor downstroke.

$$
I_{u v}=\frac{1}{m} \sum_{k=1}^{m}\left(i_{u}(t)-I_{u e}\right)^{2},
$$

where $I_{u v}$ is the current variance on the motor upstroke.

$$
I_{d v}=\frac{1}{n} \sum_{k=1}^{n}\left(i_{d}(t)-I_{d e}\right)^{2},
$$

where $I_{d v}$ is the current variance on the motor downstroke.

$$
P_{u e}=\frac{1}{m} \sum_{k=1}^{m} p_{u}(t)
$$

where $P_{u e}$ is the mean load on the motor upstroke and $p_{u}(t)$ is the real-time load on the motor upstroke.

$$
P_{d e}=\frac{1}{n} \sum_{k=1}^{n} p_{d}(t),
$$

where $P_{d e}$ is the mean load on the motor downstroke and $p_{d}(t)$ is the real-time load on the motor downstroke.

$$
P_{u v}=\frac{1}{m} \sum_{k=1}^{m}\left(p_{u}(t)-P_{u e}\right)^{2},
$$

where $P_{u v}$ is the load variance on the motor upstroke.

$$
P_{d v}=\frac{1}{n} \sum_{k=1}^{n}\left(p_{d}(t)-P_{d e}\right)^{2},
$$

where $P_{d v}$ is the load variance on the motor downstroke.

The data above are normalized using formula (12) to obtain the input vector of the fault diagnosis model $X=$ $\left[\bar{T}_{e}, \bar{F}_{o e}, \bar{F}_{c e}, \bar{I}_{u e}, \bar{I}_{d e}, \bar{I}_{u v}, \bar{I}_{d v}, \bar{P}_{u e}, \bar{P}_{d e}, \bar{P}_{d v}, \bar{P}_{d v}\right]$ :

$$
\bar{x}=\frac{x-x_{\min }}{x_{\max }-x_{\min }},
$$

where $\bar{x}$ is the normalized characteristic value, $x$ is the raw data, $x_{\max }$ is the maximum value of each data sequence, and $x_{\min }$ is the minimum value of each data sequence.

After the normalization process, the input intervals for the network are $0-1$. The input intervals help the weight and threshold value of the network enter the flat region of error curved surface.

\section{Summary of Multiple Mutation Adaptive Genetic Algorithm}

GA is an optimization algorithm with global optimization ability and includes three main processes: selection, recombination, and mutation $[15,16]$. Recombination probability $\left(P_{c}\right)$ and mutation probability $\left(P_{m}\right)$ have critical influences on the optimization ability of GA. $P_{c}$ determines the speed of new individuals as well as the speed of the search process. $P_{m}$ determines whether a new model is formed. In the optimization process, if the value of $P_{c}$ or $P_{m}$ is too large

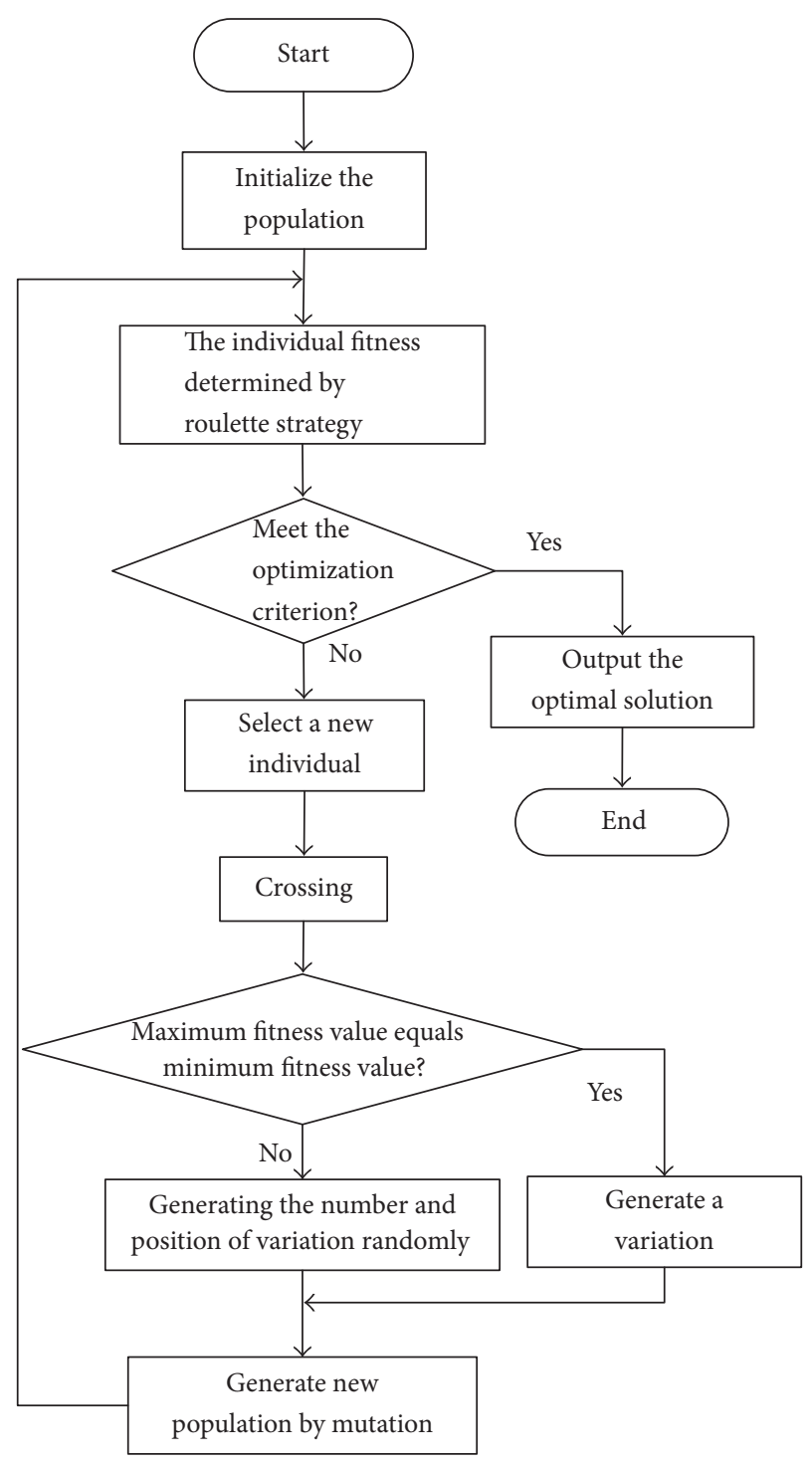

Figure 3: Algorithm flow chart of MMAGA.

or too small, the optimization ability of GA will decrease. Thus, the appropriate values of $P_{c}$ and $P_{m}$ should be selected to ensure that optimal solutions are obtained. However, MMAGA adopts the multiple genetic alterations approach [17]. The number of multiple genes mutated depends on the quality of individuals. Low fitness individuals mutate more genes, while high fitness individuals mutate less or none. MMAGA can enhance the population diversity and expand the searching space further. Its algorithm flow chart is shown in Figure 3. $P_{c}$ and $P_{m}$ are calculated using the following formulas:

$$
\begin{aligned}
& p_{c}= \begin{cases}p_{c 1} \times \frac{\hat{f}-f_{e}}{f_{\max }-f_{e}} & \widehat{f} \geq f_{e} \\
p_{c 2} & \widehat{f}<f_{e},\end{cases} \\
& p_{m}= \begin{cases}p_{m 1} \times \frac{f-f_{e}}{f_{\max }-f_{e}} & f \geq f_{e} \\
p_{m 2} & f<f_{e},\end{cases}
\end{aligned}
$$




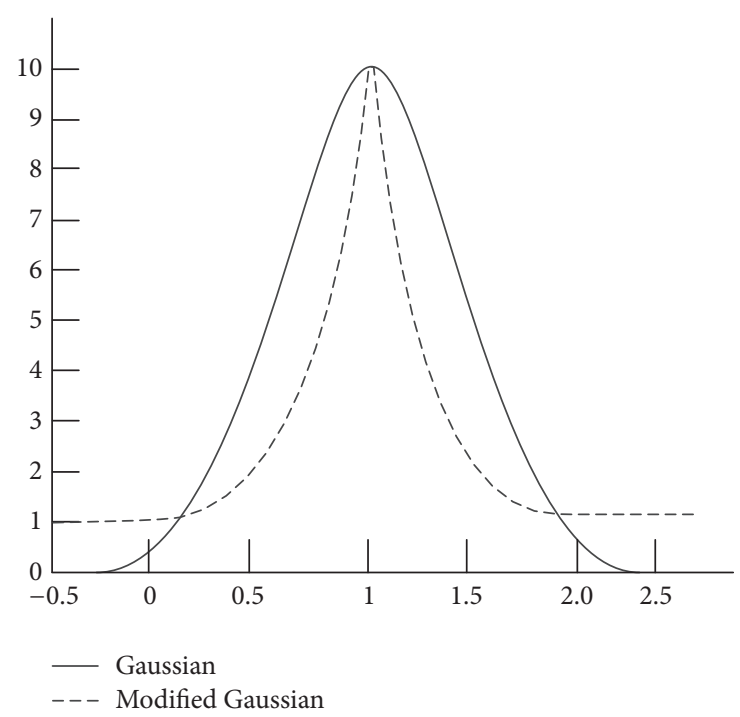

Figure 4: Characteristics of the two functions.

where $f_{\max }$ is the maximum fitness value of the population, $f_{e}$ is the mean fitness value of the population, and $\widehat{f}$ is the larger value in the restructured sides. $p_{c 1}$ and $p_{c 2}$ are hybridization constants, $f$ is the value of the individual that will mutate, and $p_{m 1}$ and $p_{m 2}$ are the mutation constants.

\section{Selection of Kernel Function of the Neural Network}

$\mathrm{BP}$ and $\mathrm{RBF}$ are the most frequently used neural networks $[18,19]$. The slow convergence speed and poor fault tolerance of the BP algorithm make it easily fall into the local optimal solution [20]. RBF algorithm has a stronger mapping ability between input and output than the BP neural network $[21,22]$. In addition, the convergence speed of the RBF algorithm is faster and the classification ability is stronger than those of the BP algorithm. However, the traditional Gaussian kernel function has some defects. This paper adopts a modified Gaussian kernel function as the kernel function of the network to improve the convergence, classification ability, diagnosis ability, and accuracy of the model further.

The form of the modified Gaussian kernel function is as follows:

$$
u_{i}=\exp \left(\frac{\sigma_{i}^{2}}{\left\|x-c_{i}\right\|^{2}+\xi^{2}}\right)
$$

where $u_{i}$ is the output of node $i$ in the hidden layer, $c_{i}$ is the center of the Gaussian kernel function, $\sigma_{i}$ is the kernel broadband variable, and $\xi$ is the displacement parameter.

The attenuation performance of the modified Gaussian kernel function is better than that of the traditional Gaussian kernel function in the data center (see Figure 4). The attenuation speed is also relatively slow when the modified Gaussian kernel function is far from the data center and tends to be a nonzero constant. Therefore, the modified Gaussian kernel function can avoid the defects of the traditional Gaussian kernel function effectively.

\section{Fault Diagnosis Model Based on the MMAGA-RBFNN for Oil Well Pump}

Oil well pumps easily present wax deposition, sand sticking, valve leakage, liquid filling dissatisfaction, pump wear, empty pumping, strong mechanical vibration, and motor shock problems. First, data from the preceding fault conditions should be collected and preprocessed to obtain the input vectors. Then, the input vectors of the fault conditions are used to train the RBF neural network and obtain its optimal weight and threshold. Finally, the fault diagnosis model is used with the optimal weight and threshold to predict new data of certain fault conditions and output the fault diagnosis results. The structure of the fault diagnosis model based on MMAGA-RBFNN is shown in Figure 5.

Although RBF neural network easily falls into local optimum and its convergence speed is slow, the MMAGA has a strong global optimization ability $[23,24]$. Therefore, this paper uses MMAGA to optimize the weight and threshold value of the RBF neural network to obtain the optimal value and increase the accuracy of the diagnosis model.

The experiment includes the following steps: (1) 300 groups of data from wax deposition, sand sticking, effect of gases, liquid filling dissatisfaction, valve leakage, and empty pumping are collected as training samples; (2) corresponding input vectors of fault conditions after preprocessing are then obtained using the training samples to train the neural network with initialized parameters; (3) selection, recombination, and mutation operations are repeated until the fault credibility satisfies the termination states; that is, it should achieve the accuracy requirement of the fault diagnosis; (4) gradient descent method is used to update the weight and threshold further until fault credibility satisfies the termination states; and (5) the optimal weight and threshold of the fault diagnosis model are obtained. The optimization flow chart of the weight and threshold value of the RBF neural network using MMAGA is shown in Figure 6.

\section{Experimental Analysis}

In the experiment, we use the oil well mock circulatory system to simulate each fault of oil well pump. This experiment platform is mainly composed of an oil well mock circulatory system, the flow control device, the oil well pump, and its control device. The experimental platform is shown in Figure 7.

On the experimental platform, six kinds of typical fault conditions (wax deposition, sand sticking, effect of gases, liquid filling dissatisfaction, valve leakage, and empty pumping) are simulated and their working condition data are recorded. Pressure and current sensors are used to collect data of the oil well pump in each fault condition. The downhole equipment is shown in Figure 8. Through the use of the oil pressure gauge, casing pressure gauge, and thermometer of the oil wellhead, the corresponding data in each fault state are also obtained. 


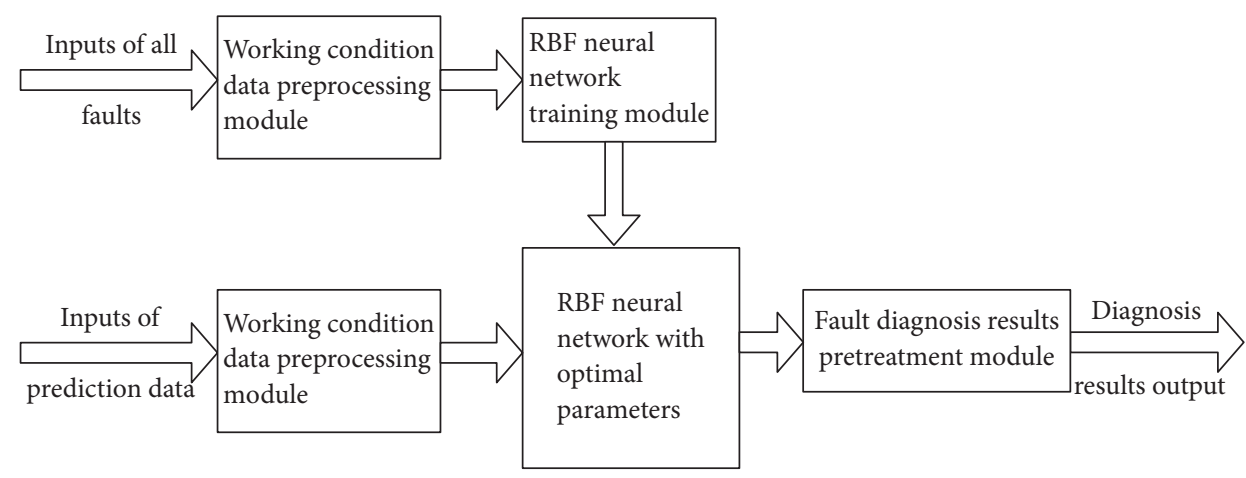

FIgURE 5: Structure of fault diagnosis model.

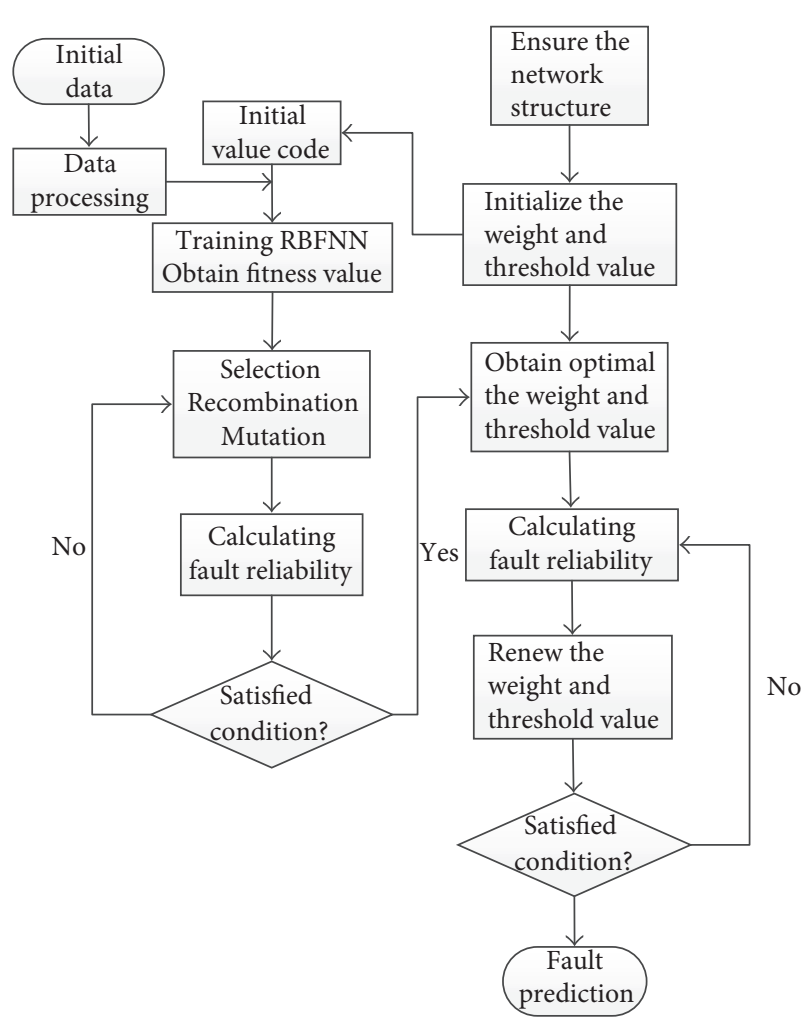

FIgURE 6: Flow chart of MMAGA optimization of RBFNN.

In the experiment, 300 groups of data from the wax deposition, sand sticking, effects of gases, liquid filling dissatisfaction, valve leakage, and empty pumping are collected as training samples. Under the same conditions, 100 groups of data are selected randomly from the faults above as test samples. The fault diagnosis model in this paper is analyzed using the training samples.

Through experimental verification, the diagnostic accuracy of the MMAGA-RBFNN fault diagnosis model is determined not only by the parameters but also by the training samples of the fault diagnosis model. The specific experimental analysis process is as follows.

First, the general performance of the model was analyzed using few training samples to train the network. After 360

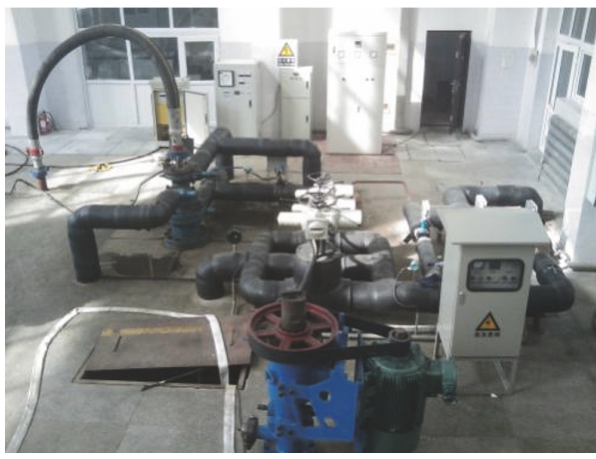

FIGURE 7: Experimental platform.

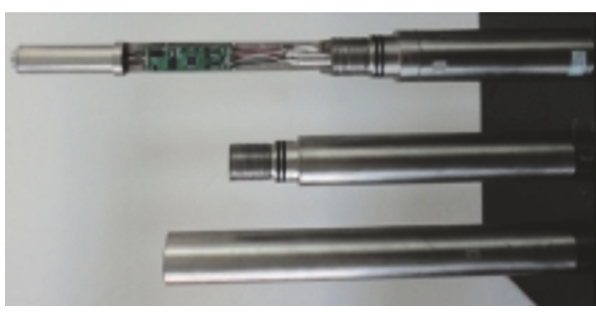

FIGURE 8: Downhole equipment.

groups of data (60 groups of data from wax deposition, sand sticking, effect of gases, liquid filling dissatisfaction, valve leakage, and empty pumping) were selected to train the network, the accuracy of the fault diagnosis was then analyzed. The accuracy of the fault diagnosis of this model can be improved only by increasing the training times because the number of training samples is small. The results of judgment rates at different number of iterations are shown in Figure 9.

When the number of iterations is low, the judgment rate is relatively low (Figure 9). In the initial period, the judgment rate increased with the number of iterations. However, when the number of iterations reaches a certain value, the growth trend of the judgment rate becomes slower or even stops. In this case, the model remains stable and overfit phenomenon occurs. In this period, although a slight rise in the judgment rate occurred, the study process is meaningless because the 


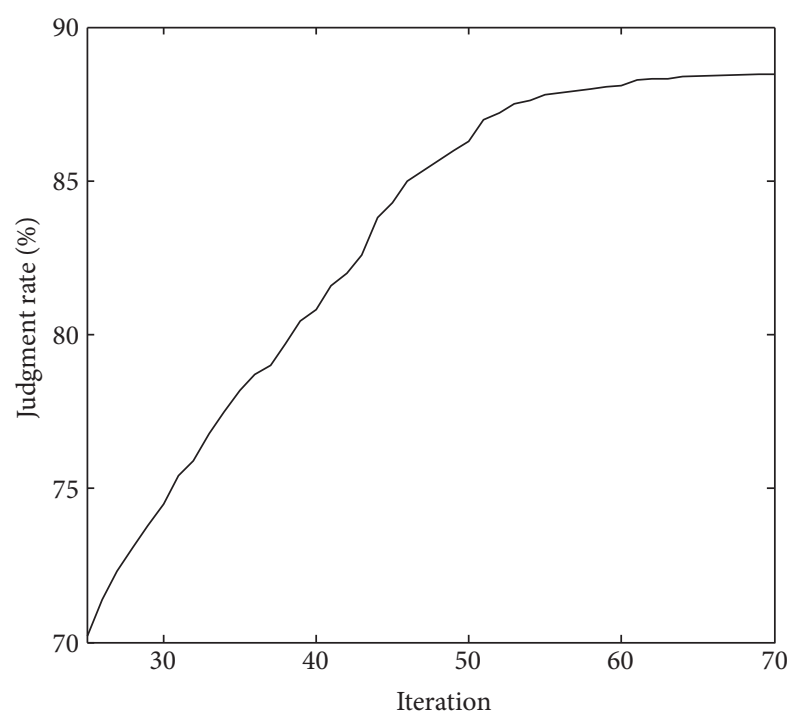

Figure 9: Curve of judgment rate.

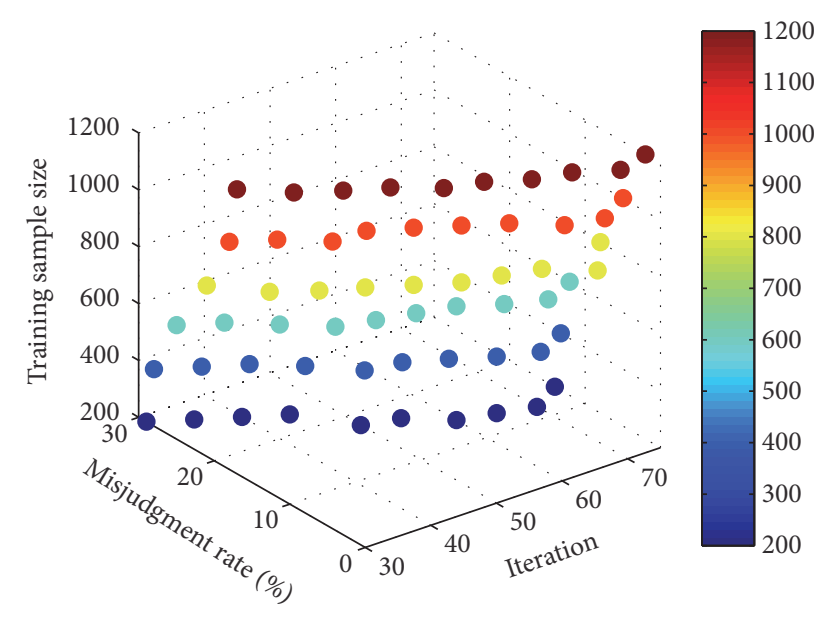

Figure 10: Misjudgment rates of test data.

diagnosis model could not be extended to the testing data accurately.

Increasing the number of training samples can prevent the occurrence of overfit phenomenon and increase the judgment rate of the model effectively. Therefore, 200-1200 groups of training data were selected to test the misjudgment rates at different numbers of iterations. The results are shown in Figure 10.

The comprehensive misjudgment rates decreased when the number of training samples increased (Figure 10). The comprehensive misjudgment rate can be controlled at less than $4 \%$ when the number of training samples is over 1000 . Therefore, the diagnostic ability of the developed model is high. Among above 6 fault conditions, though the sand sticking and the wax deposition are easily misjudged, other types of faults can be accurately diagnosed.

The judgment rates at different training samples are shown in Figure 11 by comparing the MMAGA-RBFNN diagnosis model with the GA-RBFNN model. When the number

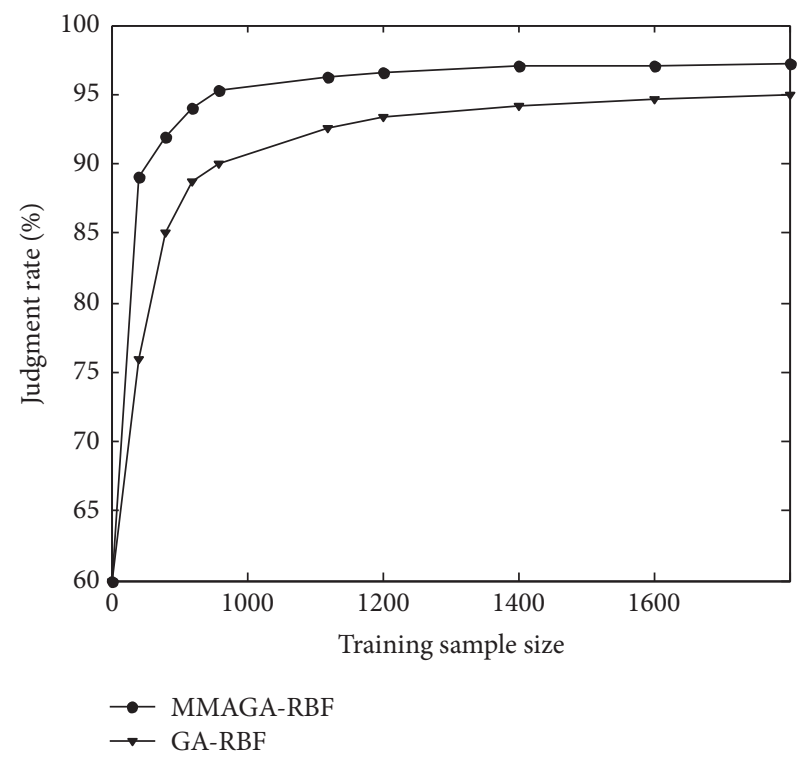

FIGURE 11: Judgment rates curves of two models.

of training samples is small, the judgment rates of the two models are relatively low (Figure 11). However, the judgment rate of MMAGA-RBFNN model is significantly $5 \%$ higher on average than that of the GA-RBFNN model when the number of training samples increases. Obtaining many training samples is costly. Therefore, increasing the training samples is not a feasible solution to improve the judgment ability of the diagnosis model.

\section{Conclusion}

The advantages of the fault diagnosis model proposed in this paper based on the MMAGA-RBFNN for oil well pump are as follows.

(1) The judgment rate of the developed model increases with the number of training samples increase. The judgment rate can reach over $96 \%$ when the number of training samples is over 1000. This shows that this model has a very high judgment rate when the training samples are relatively fewer. Compared to other models, the model proposed in this paper uses fewer training samples and higher accuracy and reduces the cost and workload.

(2) The developed model effectively avoids the defects of traditional Gaussian kernel function, improves convergence and classification ability, and enhances the diagnosis ability and accuracy. In addition, the developed model's fault diagnosis rate increased by $5 \%$ on average compared to the previous model.

(3) The generalization ability of the developed model is strong and can meet the working condition characteristics of oil well pump. The developed model can satisfy the diagnostic accuracy of faults for the oil well pump. We can deal with the oil well pump faults in a timely manner because it can forecast the occurrence of faults accurately. Thus, the work reliability of oil well pump can be improved. 
In general, the MMAGA-RBFNN model satisfies the requirements of generalization and accuracy for the fault diagnosis of the oil well pump. This model can predict the fault conditions of oil well pumps accurately and improve the reliability and economic benefits.

\section{Conflicts of Interest}

The authors declare that they have no conflicts of interest.

\section{References}

[1] I. I. Abdalla, T. Ibrahim, and N. B. Mohd Nor, "Development and optimization of a moving-magnet tubular linear permanent magnet motor for use in a reciprocating compressor of household refrigerators," International Journal of Electrical Power and Energy Systems, vol. 77, pp. 263-270, 2016.

[2] E. A. Dil, M. Ghaedi, A. Asfaram, F. Mehrabi, A. A. Bazrafshan, and A. M. Ghaedi, "Trace determination of safranin $O$ dye using ultrasound assisted dispersive solid-phase micro extraction: Artificial neural network-genetic algorithm and response surface methodology," Ultrasonics Sonochemistry, vol. 33, pp. 129-140, 2016.

[3] G. Chun and W. Zhenglin, Proficient in MATLAB optimization, Electronic Industry press, Beijing, China, 3rd edition, 2014.

[4] X. Guan, Y. Zhu, and W. Song, "Application of RBF neural network improved by peak density function in intelligent color matching of wood dyeing," Chaos, Solitons and Fractals, vol. 89, pp. 485-490, 2016.

[5] H. B. Huang, R. X. Li, X. R. Huang, T. C. Lim, and W. P. Ding, "Identification of vehicle suspension shock absorber squeak and rattle noise based on wavelet packet transforms and a genetic algorithm-support vector machine," Applied Acoustics, vol. 113, pp. 137-148, 2016.

[6] A. Jiang, Y. Li, and C. Xue, "Study of PSO-RBF Neural Network in Power System Load Prediction," in Proceedings of the 2015 2nd International Conference on Electrical, Computer Engineering and Electronics, Shunhe, China, June 2015.

[7] J.-W. Jung, V. Q. Leu, D. Q. Dang, H. H. Choi, and T. H. Kim, "Sliding mode control of SPMSM drivers:an online gain tuning approach with unknown system parameters," Journal of Power Electronics, vol. 14, no. 5, Article ID JPE 14-5-19, pp. 980-988, 2014.

[8] L. Liyi, T. Yongbin, and L. Jiaxi, "Application of the multiple population genetic algorithm in optimum design of air-core permanent magnet linear synchronous motors," in Proceedings of the CSEE, vol. 33, pp. 69-77, 2013.

[9] X. Li, L. Zhao, L. Wei et al., "Deepsaliency: multi-task deep neural network model for salient object detection," IEEE Transactions on Image Processing, vol. 25, no. 8, pp. 3919-3930, 2016.

[10] B. Liu, X. Huang, and J. Li, “Optimal sizing of distributed generation in a typical island microgrid with time-shifting load," Zhongguo Dianji Gongcheng Xuebao/Proceedings of the Chinese Society of Electrical Engineering, vol. 34, no. 25, pp. 4250-4258, 2014.

[11] J. Liu, L. Huang, H. Yu, C. Wen, and W. Zhong, "Study on the characteristics of a novel six-phase fault-torrent linear permanent magnet machine for linear oil pumping," IEEE Transactions on Applied Superconductivity, vol. 24, no. 3, 2014.

[12] H. Melo and J. Watada, "Gaussian-PSO with fuzzy reasoning based on structural learning for training a Neural Network," Neurocomputing, vol. 172, pp. 405-412, 2016.
[13] M. Omura, T. Shimono, and Y. Fujimoto, "Development of semicircular tubular coreless linear motor and its motion control," Electrical Engineering in Japan (English translation of Denki Gakkai Ronbunshi), vol. 195, no. 4, pp. 50-63, 2016.

[14] P. Garanayak and G. Panda, "Harmonic elimination and reactive power compensation with a novel control algorithm based active power filter," Journal of Power Electronics, vol. 15, no. 6, pp. 1619-1627, 2015.

[15] L. Shi, J. Tang, P. Wang, L. Han, and X. Zhang, "Stator fault diagnosis of induction motors using the optimal wavelet tree and improved BP neural network," Diangong Jishu Xuebao/ Transactions of China Electrotechnical Society, vol. 30, no. 24, pp. 38-45, 2015.

[16] Y. Tang, H. Gao, and J. Kurths, "Multiobjective identification of controlling areas in neuronal networks," IEEE/ACM Transactions on Computational Biology and Bioinformatics, vol. 10, no. 3, pp. 708-720, 2013.

[17] Y. Tian, Z. Wang, C. Lu, and Z. Wang, "Bearing diagnostics: A method based on differential geometry," Mechanical Systems and Signal Processing, vol. 80, pp. 377-391, 2016.

[18] W. Bo1, Z. Haisen1, L. Heming1, and L. Shoujun, "Design and applications of the testing system with dynamic load for beam pumping motor," in Proceedings of the CSEE, vol. 34, pp. 34883495, 2014.

[19] D. Wang, "Sensitivity analysis of structural response to position of external applied load: in plane stress condition," Structural and Multidisciplinary Optimization, vol. 50, no. 4, pp. 605-622, 2014.

[20] X. Niu, J. Wang, and Z. Ren, "A fault diagnosis based on combination model of VPRS and PSO-BP neural network," Chemical Engineering Transactions, vol. 46, pp. 319-324, 2015.

[21] Y. Xie, W. Xiong, D. Zhuo, and X. Wang, "Drawbead geometric parameters using an improved equivalent model and PSO-BP neural network," Proceedings of the Institution of Mechanical Engineers, Part L: Journal of Materials: Design and Applications, vol. 230, no. 4, pp. 899-910, 2016.

[22] D. Yu, Y. Zhang, H. Bian, X. Wang, and W. Qi, "A new diagnostic method for identifying working conditions of submersible reciprocating pumping systems," Petroleum Science, vol. 10, no. 1, pp. 81-90, 2013.

[23] D. Yu, S. Deng, Y. Zhang, and W. Qi, "Working condition diagnosis method based on SVM of submersible plunger pump," Diangong Jishu Xuebao/Transactions of China Electrotechnical Society, vol. 28, no. 4, pp. 248-284, 2013.

[24] E. Zhang, L. Hou, C. Shen, Y. Shi, and Y. Zhang, "Sound quality prediction of vehicle interior noise and mathematical modeling using a back propagation neural network (BPNN) based on particle swarm optimization (PSO)," Measurement Science and Technology, vol. 27, no. 1, Article ID 015801, 2015. 


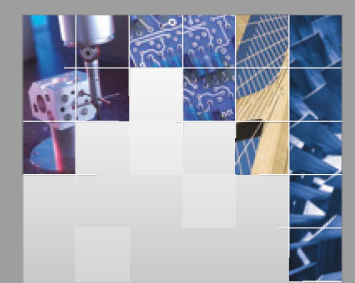

\section{Enfincering}
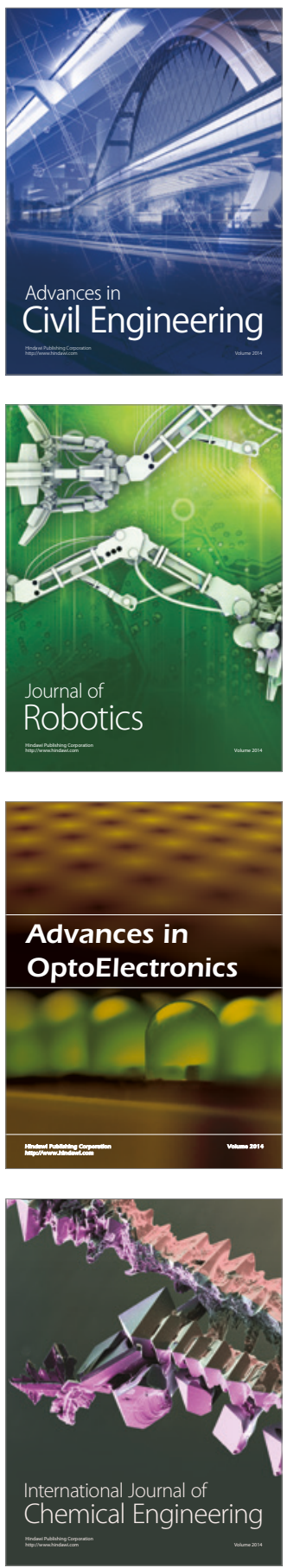

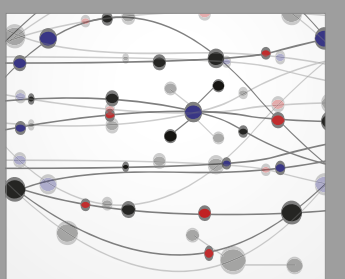

The Scientific World Journal

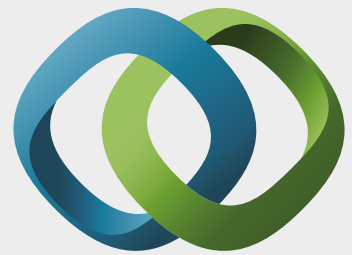

\section{Hindawi}

Submit your manuscripts at

https://www.hindawi.com
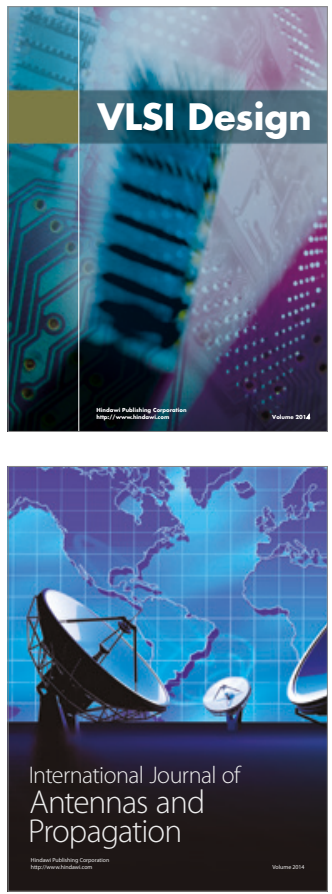

\section{Rotating}

Machinery
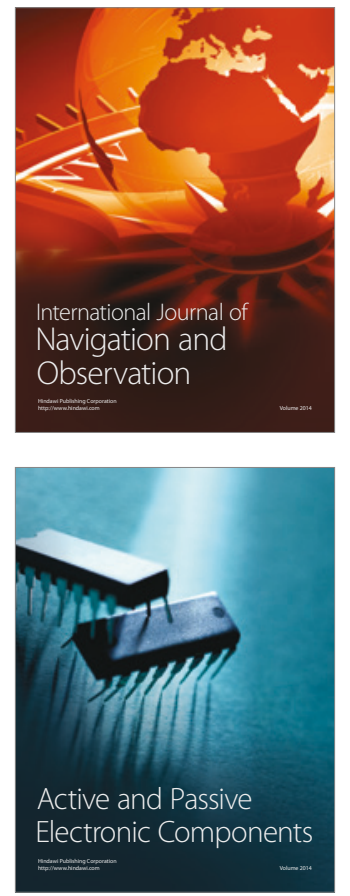
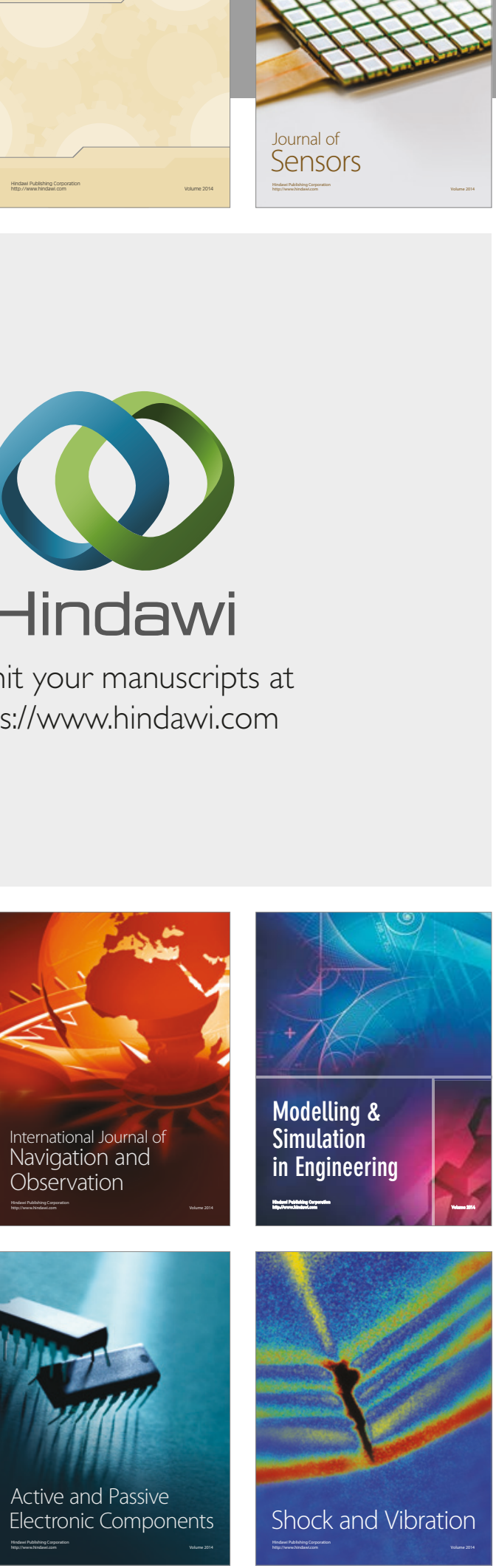
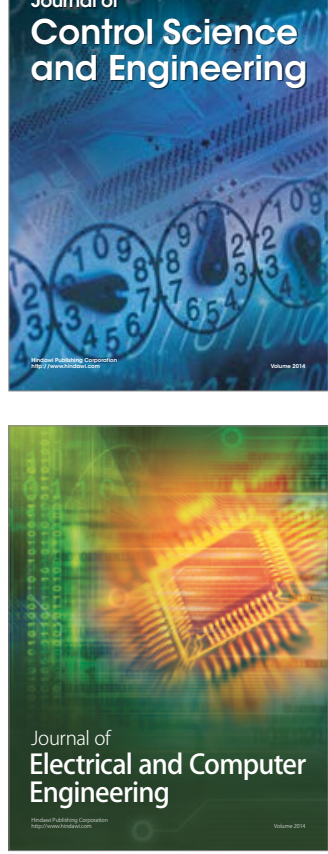

Distributed

Journal of

Control Science

and Engineering
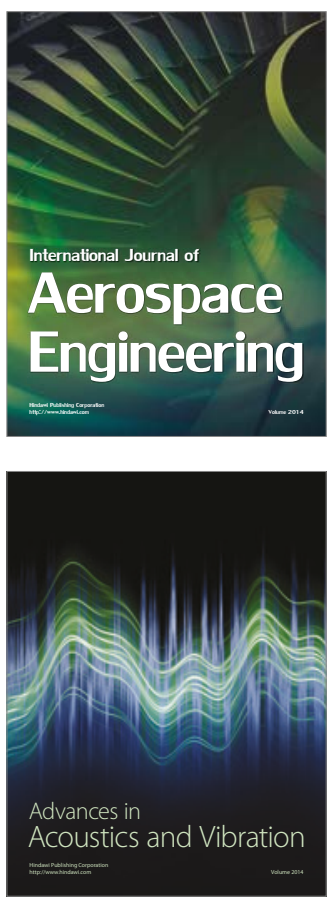

Sensor Networks 